\title{
Trajectory Mining, Representation and Privacy Protection
}

\author{
Jiaxin Ding \\ Supervised by Jie Gao \\ Stony Brook University \\ jiading@cs.stonybrook.edu
}

\begin{abstract}
The maturing of mobile devices and systems provides an unprecedented opportunity to collect a large amount of real world human motion data at all scales. The rich knowledge contained in these data sets is valuable. In this analysis, we first study information dissemination through the physical contacts learnt from the data sets. Also, we propose the topological representation of trajectories, specially the homotopy types, which record how trajectories pass around certain regions of interest in the domain. Thereafter, as various types of personally sensitive information can be revealed from the published data sets, we present a simple algorithm, mix-and-match, for the type of applications that requires a faithful distribution at any temporal snapshot but does not require geometric shape of each individual to be preserved.
\end{abstract}

\section{INTRODUCTION}

The past decade has witnessed the rapid development of techniques of obtaining human trajectories. In outdoor settings, GPS units have become a norm on smartphones and vehicles. Mobile phone records routinely collected by wireless service provider can also help to pin down locations within a small margin of error. For indoor scenarios, sensing modalities ( $\mathrm{RF}$ signals, infrared etc.) make real time tracking a reality. All the above techniques provide us an opportunity to collect a large amount of trajectories. In this paper, we will first show the discoveries of information dissemination patterns using the trajectories data sets. Then, we will provide some ideas of the topological representation of such trajectories. Finally, we show algorithms to protect the trajectory privacy.

\section{INFORMATION DISSEMINATION THR- OUGH PHYSICAL CONTACTS}

Social contacts refer to people's engagement in social activities and information exchange. Information dissemination through social contacts can be potentially very fast, Permission to make digital or hard copies of part or all of this work for personal or classroom use is granted without fee provided that copies are not made or distributed for profit or commercial advantage and that copies bear this notice and the full citation on the first page. Copyrights for third-party components of this work must be honored For all other uses, contact the owner/author(s).

SIGSPATIAL PhD Workshop'15, November 03-06 2015, Bellevue, WA, USA

http://dx.doi.org/10.1145/2855680.2855839

Copyright 2015 ACM 978-1-4503-3980-3/15/11 demonstrating the power of "word of mouth" [8]. This is partly attributed to the network structure, i.e., the small world property that any two individuals are connected through a small number of hops [10]. Thus, if each social contact helps to disseminate the information, within a small number of hops the message reaches the entire network. This fact serves as one of the foundations of online social networking platforms and promises of viral marketing through that.

Physical contacts refer to individuals staying at the same location at the same time. Physical co-location presence, on the other hand, is much less understood and exploited for spreading information. We argue that enabling selective and effective information exchanges through physical contacts can be extremely useful for certain applications and in some cases such benefits can be hardly achieved by other means. Take the example of crime scene recovery. People who were at the crime scene have valuable first-hand information for timely locating the suspects, witness identification and crime investigation. Many other application scenarios, such as locating missing persons, identifying correlated terrorism activities, fall in the same category. Existing support of social media, which mainly focuses on social contacts, does not capture the physical co-location of individuals/vehicles without social connections and is therefore of limited help. We need to understand and harvest the potential information dissemination opportunities through physical contacts to effectively address such challenges. In this work, we focus on information dissemination through physical contacts and examine its characteristics and potentials.

As vehicular ad hoc network has been developing fast and is going to come into use in the near future. We focus the analysis on the "physical contacts" of vehicles, namely, two vehicles are within communication range. For this reason, we choose to use GPS traces of vehicles. When two vehicles are within close proximity, short-range communication primitives, such as IEEE 802.11p standard for Wireless Access in the Vehicular Environment (WAVE), can be adopted for opportunistic information exchanges.

In our study, we have analyzed a number of large-scale trajectory data from taxis in multiple cities. One of the data sets includes 9,386 taxis in a large city over a period of 24 hours. We understand that taxi mobility patterns differ from mobility patterns of private vehicles. But getting detailed GPS trajectories for a large number of private vehicles is much more challenging due to privacy concerns. The GPS traces from taxis thus appear to be the best data set for 
our objective. In addition, taxis represent a decent fraction of vehicles in any large city. Opportunities enabled by taxi mobility for disseminating information provide a conservative lower bound on what might be achieved when private vehicles also voluntarily participate.

We consider the setting in which each vehicle starts with a unique message. When two vehicles are within direct communication range the two vehicles exchange all data messages they have. We examine how fast such data messages spread in the vehicle system and what the critical system parameters are that affect the efficiency and delay of such dissemination.

We discover that the real world mobility patterns for taxis are highly irregular and non-random. The mean speed of the vehicles shows great fluctuation with clear daily patterns. Despite the irregularities of the motion patterns, the natural vehicular mobility has surprisingly good support for information dissemination in the vehicular system. We discovered two universal properties in the data sets for multiple cities. First, the messages travel in a "small-world" manner. We look at the number of "hops" a message initiated at vehicle $i$ travels until it first arrives at a vehicle $j$, for all pairs of node $i, j$. The distribution for this measure gives a medium of 9 for $10 \mathrm{~K}$ vehicles, which is surprisingly small considering the scale of the system. We also look at the number of messages exchanged during an effective exchange opportunity, which shows a clear power-law distribution. That is, a few of the exchanges manage to exchange a large quantity of data. The existence of these highly effective exchanges probably explains the small world property. The last observation is regarding the "popularity" of different participating vehicles, defined as the number of physical contacts one vehicle has with all others. In most social network settings, the popularity is measured by the degree of the nodes, say the number of friends, the number of in-links or followers. This distribution often shows a power law distribution. There are "hubs" with high degree whose removal substantially hurt the network connectivity. In the vehicular setting, removing the vehicles whose physical contact counts rank at the top $20 \%$ does not show any substantial difference for information dissemination. The fact that there are no "hubs" implies certain robustness of information dissemination by the natural mobility patterns.

We would like to remark that these observations confirms that fast information dissemination through physical contacts is promising. The fact that the messages travel with a small world property shows effectiveness and efficiency, especially when aggregation is adopted with message passing. The spatial temporal pattern of message dissemination also coincides with the typical user request pattern - that users are often more interested in things happening in a close proximity and luckily such data indeed becomes available faster.

\section{TOPOLOGICAL REPRESENTATION}

One of the fundamental issues is how to represent trajectories. Mathematically a motion trajectory is a time-stamped $2 \mathrm{D}$ curve that is often represented by a polygonal curve. That is, a trajectory is sequence of points $(x, y, t)$ meaning that the target is at location with coordinates $(x, y)$ at time $t$. And it is common practice to perform linear interpolation between adjacent data points to complete the sequence of points into a polygonal curve. This is denoted the geometric representation of a trajectory. In some of the anonymization schemes in the literature, each data point $(x, y, t)$ is coarsened to a region $(R, t)$ meaning that the target is inside $R$ at time $t$. The size of $R$ describes the resolution of data.

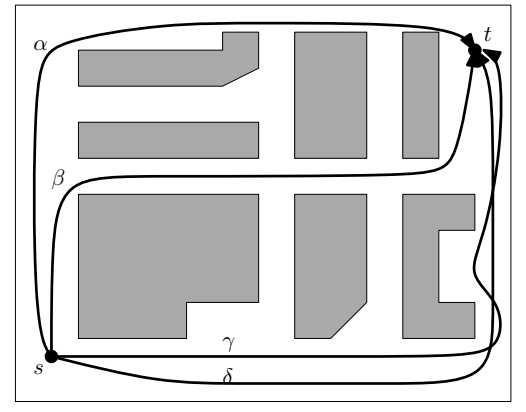

Figure 1: The network has a few holes/obstacles shaded. Paths $\alpha, \beta, \gamma$ all have different homotopy type. $\gamma$ and $\delta$ are homotopy equivalent.

In this analysis, we will also investigate the topological representation of a trajectory. We argue that there are benefits of doing so. In a topological representation, we represent a trajectory by how it moves around other specified obstacles in the same domain. These obstacles are often real obstacles (city blocks, buildings, lakes, or furniture and walls) or important landmarks. Take the example in Fig. 1. We are showing a toy example of some metropolitan area in which the roads are surrounding city blocks. Vehicles trajectories naturally stay in between these blocks. Most of real world trajectories follow near shortest paths, and do not take unnecessary detours. Under this assumption, if we know the source and destination of a trajectory and how it goes around the blocks, we can reconstruct a path that is similar to the ground truth. For example, $\delta$ might be a reconstructed path for $\gamma$. It is true that this topological information is not sufficient to pin down the exact geometry of the specific path, e.g., we cannot tell which lane the vehicle has taken. But a fair amount of high-level geometric information is well preserved such as the sequence of roads followed by the vehicles, which is enough for many applications that only care about the coarse grained geometry. Indeed, to understand the general pattern in a group of trajectories, we precisely need to filter out the unnecessarily fine geometric details and keep only the most prominent features of the trajectories such that one can define movement patterns and behaviors including flocking, convoys, herds, leadership, commuting, and various others. All trajectories that share the same source/destination and tour around obstacles in the same pattern are naturally grouped in the same bucket.

The mathematical definition of how a trajectory goes around obstacles is denoted its homotopy type. Let us look at the example in Fig. 1. Observe that the paths $\alpha, \beta, \gamma$ are all different in a global sense. One can not deform $\alpha$ to $\beta$ unless it jumps over some obstacles. However, paths $\gamma$ and $\delta$ are only different in a local manner. One can deform $\gamma$ to $\delta$ smoothly through some local changes. This difference is characterized by the homotopy type of a path. Two paths are homotopy equivalent if one can be smoothly deformed to the other. In other words, the cycle formed by two path of the same homotopy type can be shrunk to zero. In theory the number of homotopy types is infinite, since one can loop around a hole infinitely many times. But in practice only a finite number of homotopy types are of interest. In the topological repre- 
sentation, the resolution is controlled by how the obstacles are placed. For example, if we take all the trajectories as an arrangement of curves and place an artificial obstacle in each face of the planar decomposition, we can ensure that all trajectories are homotopically different - allowing maximum level of differentiation.

\section{TRAJECTORY PRIVACY PROTECTION}

Protecting location privacy was brought to the attention of the research community a few years ago [3]. Plenty of work has been conducted to protect the privacy of a single location, when the user submits a query to location-based services (LBS) on mobile phones. An adversary that compromises the LBS server can infer private information about the user. In this setting, protection of privacy is characterized into two different yet related types: query privacy, e.g., whether an adversary can identify the user who issued the query, and location privacy, e.g., whether a user can be accurately located. Most privacy protection algorithms use location perturbation and obfuscation methods.

For query privacy, the most common approach is to use $k$-anonymity measure and design 'cloaking boxes' [6]. Users submit their queries to a trusted server called the anonymizer who group spatio-temporally similar queries into a box of at least $k$ queries and submit the whole box of queries together to the LBS server. The sender's info is removed when the anonymizer submits the box. By this way, the query sender is indistinguishable from the $k-1$ other query users in the same box.

For location privacy, one common metric used is location entropy, which characterizes the uncertainty of the location information an adversary can extract from LBS queries. Most methods use perturbations of the true locations to confuse the adversary (for example, see [7]). Notice that the reported location cannot be too far away from the true location as otherwise the location based query will become useless. There is a tradeoff of location privacy versus query utility.

Protecting the privacy of the trajectory data is not investigated as much. This is partly due to the unique geometric structures in the trajectory data that call for novel geometric algorithms. Previous work is along the following directions.

When mobile phones continuously issue location based services, applying a spatial cloaking at each snapshot is not enough to guarantee $k$-anonymity for trajectories. Thus temporal spatial cloaking boxes are created $[5,12]$. The downside is that the cloaking box becomes huge after a long time period, leading to high computational cost and low query accuracy.

A different idea [2] introduces 'mixed-zones', a geometric region such that users entering the zone change to a pseudonym and do not send LBS queries. If the mixed zone has $k$ users, the probability of each user leaving the zone along each exit is the same and each user spends a random duration of time inside the zone, the set of users are $k$-anonymized. In the case of protecting privacy during publication of trajectories to a third party, two ideas have been mainly used: clustering based [1] and generalization based [11]. In clustering based approach, the trajectory data is clustered into groups of $k$ co-located trajectories within the same time period to form a $k$-anonymized aggregated trajectory. The limitation of this approach is the strong requirement that such clusters can be found in the data set. In addition, many applications require atomic trajectories and are not ready to take aggregated trajectories. In generalization based approach, each trajectory is first transformed into a sequence of $k$-anonymized regions. Then for each trajectory, the algorithm uniformly select $k$ atomic points in each anonymized region and link a unique atomic point from each region to reconstruct $k$ trajectories. Performance of this approach can be evaluated in two aspects - how big the anonymized regions are and how much the altered data change the utility of this data set.

In [9], dummies with movement patterns similar to those of real users are generated. Mixture of queries from both true users and dummies are sent to the LBS to confuse the adversary. One issue to be aware of for the inclusion of dummies is how much the added noises alter the properties of the data set and consequently decrease the data utility.

\subsection{Mix-And-Match Algorithm}

Given a set of time-stamped paths, we want to keep the data points at each snapshot to be exactly the same, but we have the freedom to change how they are linked to form trajectories. Our idea for anonymizing the trajectory data is to mix the IDs of the trajectories randomly, when two trajectories intersect (meet at the same location at the same time). Specifically, when a trajectory with current ID $i$ meets a trajectory $j$, we swap the two segments out of the junction randomly. The real trajectory of ID $i$ now carries ID $j$ with probability $p$. See Figure 2 for an example. After all swaps, we collect all the segments with the same ID together as a newly generated trajectory set.

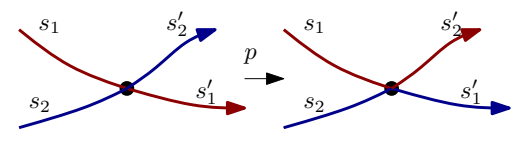

Figure 2: We use red and blue to denote two trajectories with different IDs. The figure on the left is the original trajectory and the one on the right is the published trajectory. Originally, the red trajectory consists of $\left(s_{1}, s_{1}^{\prime}\right)$ and the blue combines $\left(s_{2}, s_{2}^{\prime}\right)$. If the swap happens at the junction point with probability of $p$, the newly generated red trajectory changes to $\left(s_{1}, s_{2}^{\prime}\right)$ while the blue turns to be a combination of $\left(s_{2}, s_{1}^{\prime}\right)$.

The random mixing neither change any location data nor does it introduce any dummy points. The distribution at any snapshot is faithfully preserved. What is changed is how the segments, defined as pieces of trajectories between two junction points, are glued to one another. After the anonymization process, a trajectory defined as the path following the same ID is possibly composed of pieces of different trajectories in the original data set.

To evaluate the effectiveness of this algorithm, we wish to understand what properties of the initial trajectories, both individually and as a whole set, have been changed. First we observe that the association of IDs with trajectory segments should be sufficiently 'mixed'. In the extreme case, if the trajectories have no intersections, then nothing is going to happen with our mix-and-match algorithm. Therefore the first requirement is that the intersection pattern of the trajectories are sufficiently 'complex'. We denote this as the combinatorial diversity of the trajectories. Second, only the mixing of the association of IDs is not enough to ensure that the unique signatures of the trajectories are erased. In 
an extreme example, consider $n$ trajectories that all follow almost exactly the same road, mixing with each other a million times along the way. The resulted trajectories will have their IDs sufficiently mixed, but the output trajectories are geometrically almost the same as before. Therefore, we must also make sure that the geometric features of the trajectories are sufficiently altered. This boils down to characterization of how much geometric diversity there is in the initial trajectories and how such diversity aids in erasing old patterns after the mixing step.

In the following we provide analysis on the two aspects on basic parameters of the input trajectory data. In the discussion we will assume that the adversary has the entire data set and the knowledge of our algorithm but not the random choices made for the intersections. We then discuss how the adversary could recover the combinatorial or geometric properties of the data set.

Combinatorial Diversity. Assume we have $n$ vehicles. At the end of each time slot $(t)$, we have a $n \times n$ matrix $X(t)$, where $X_{j i}(t)$ represents the probability, at time $t$, the segment originally belonging to $j$ now carries the ID $i . X_{i}(t)$ is the $i$ th column of $X$, representing the probability distribution of over all trajectories carrying ID $i$ at time $t$. We define the combinatorial diversity for ID $i$ as

$$
\frac{\left\|X_{i}(t)-X_{i}(0)\right\|}{\left\|X_{i}(0)\right\|}
$$

where $X_{i}(0)=e_{i}$. The maximum value of the combinatorial diversity is $\sqrt{2}$ with $X_{i}(t)=e_{j}$ for all $j \neq i$. It measures the difference between the current probability distribution for segments carrying ID $i$ and the initial distribution. The more it deviates from the initial distribution, the harder it is to re-identify. For any given set of trajectories one can evaluate the combinatorial diversity as mentioned above.

Geometric Diversity. When the combinatorial diversity is enough to ensure that the IDs are mixed enough, we will examine the geometric diversity in the data set. On this level, the trajectories obtained through the mix-and-match process should be sufficiently far away from the original one. One way to evaluate this is by examining the mean Euclidean distances between the different trajectories in the output as well as the difference of the trajectories with the same ID before and after the mixing process. $d_{i j}(t)$ is the distance between the original $i$ th trajectory and $j$ the trajectory at time $t$. The geometric diversity of the $i$ th trajectory at time $t$ is defined as:

$$
\begin{aligned}
& E\left(d_{j i} \mid \text { the } j \text { th trajectory is carrying ID } i \text { at } t\right) \\
= & \sum_{j=1}^{n} d_{j i}(t) X_{j i}(t)
\end{aligned}
$$

In our work, we have addressed the following issues: 1) In theory, under some modest assumption of mobility, the combinatorial diversity can be sufficiently large with high probability. 2)Testing in real motion trajectories, there are sufficient co-location opportunities. And such junctions will lead to a sufficiently combinatorial and geometric diversity. 3) We would like to characterize the attacking model of adversary, with which we can test the probability our algorithm to be attacked.

\section{CONCLUSION}

In this work, we have illustrated some discoveries with spatial data, introduced a new idea of topological representation of trajectories and proposed a privacy protection method.

\section{REFERENCES}

[1] O. Abul, F. Bonchi, and M. Nanni. Never walk alone: Uncertainty for anonymity in moving objects databases. In Data Engineering, 2008. ICDE 2008. IEEE 24th International Conference on, pages 376-385, April 2008.

[2] A. Beresford and F. Stajano. Location privacy in pervasive computing. Pervasive Computing, IEEE, 2(1):46-55, Jan 2003.

[3] A. J. Blumberg and P. Eckersley. On locational privacy, and how to avoid losing it forever. EFF whitepaper, https://www.eff.org/files/eff-locational-privacy.pdf, 2009.

[4] S. Boyd, A. Ghosh, B. Prabhakar, and D. Shah. Randomized gossip algorithms. Information Theory, IEEE Transactions on, 52(6):2508-2530.

[5] C.-Y. Chow and M. F. Mokbel. Enabling private continuous queries for revealed user locations. In Proceedings of the 10th International Conference on Advances in Spatial and Temporal Databases, SSTD'07, pages 258-273, Berlin, Heidelberg, 2007. Springer-Verlag.

[6] M. Gruteser and D. Grunwald. Anonymous usage of location-based services through spatial and temporal cloaking. In Proceedings of the 1st International Conference on Mobile Systems, Applications and Services, MobiSys '03, pages 31-42, New York, NY, USA, 2003. ACM.

[7] B. Hoh and M. Gruteser. Protecting location privacy through path confusion. In Security and Privacy for Emerging Areas in Communications Networks, 2005. SecureComm 2005. First International Conference on, pages 194-205, Sept 2005.

[8] D. Kempe, J. Kleinberg, and É. Tardos. Maximizing the spread of influence through a social network. In Proceedings of the ninth ACM SIGKDD international conference on Knowledge discovery and data mining, pages 137-146. ACM, 2003.

[9] H. Kido, Y. Yanagisawa, and T. Satoh. An anonymous communication technique using dummies for location-based services. In Pervasive Services, 2005. ICPS '05. Proceedings. International Conference on, pages 88-97, July 2005.

[10] S. Milgram. The small world problem. Psychology Today, (1), 1967

[11] M. E. Nergiz, M. Atzori, Y. Saygin, and B. Güç. Towards trajectory anonymization: A generalization-based approach. Trans. Data Privacy, 2(1):47-75, Apr. 2009.

[12] T. Xu and Y. Cai. Location anonymity in continuous location-based services. In Proceedings of the 15th Annual ACM International Symposium on Advances in Geographic Information Systems, GIS '07, pages 39:1-39:8, New York, NY, USA, 2007. ACM. 